\title{
Bose-Einstein correlations of neutral gauge bosons in $p p$ collisions
}

\author{
G.A. Kozlov \\ Bogolyubov Laboratory of Theoretical Physics \\ Joint Institute for Nuclear Research, \\ Joliot Curie st., 6, Dubna, Moscow region, 141980 Russia
}

\begin{abstract}
The theory for Bose-Einstein correlations in case of neutral gauge bosons in $p p$ collisions at high energies is presented. Based on quantum field theory at finite temperature the two-particle Bose-Einstein correlations of neutral gauge bosons are carried out for the first time. As a result, the important parameters of the correlation functions can be obtained for the $Z^{0} Z^{0}$ pairs. The correlations of two bosons in 4-momentum space presented in this paper offer useful and instructive complimentary viewpoints to theoretical and experimental works in multiparticle femtoscopy and interferometry measurements at hadron colliders.
\end{abstract}




\section{Introduction}

An investigation of the space-time extension or even squeezing of particle sources via the multiparticle quantum-statistics correlation in high energy interactions is still attract the attention of physical society in both experiment and theory. Over the past few decades, a considerable number of successful studies have been done in this direction [1]. It is well understood that the studies of correlations between produced particles, the effects of coherence and chaoticity, an estimation of particle emitting source size play an important role in high energy physics.

By studying the Bose-Einstein correlations (BEC) of identical particles (we mean like-sign charge particles and the neutral charge ones), it is possible experimentally to determine the time scale and spatial region over which particles do not have the interactions. Such a surface is called as decoupling one. In fact, for an evolving system such as $p p$ collisions, it is not really a surface, since at each time there is a spread out surface due to fluctuations in the last interactions, and the shape of this surface evolve even in time. The particle source is not approximately constant because of energy-momentum conservation constraint.

More than half a century ago Hanbury-Brown and Twiss [2] used BEC between photons to measure the size of distant stars. In the works $[3,4]$, the master equations for evolution of thermodynamic system that can be created at the final state of a high multiplicity process were established. The equations have the form of the field operator evolution equation (Langevin-like [5]) and allows one to gain the basic features of the emitting source space-time structure. In particular, it has been conjectured and further confirmed that the size of the emitting source through BEC is strongly affected by non-classical off-shell effect.

The shapes of BEC function were experimentally established in the LEP experiments ALEPH [6], DELPHI [7] and OPAL [8], and ZEUS Collaboration at HERA [9], which also indicated a dependence of the measured correlation radius on the hadron $(\pi, K)$ mass. The results for $\pi^{ \pm} \pi^{ \pm}$and $\pi^{ \pm} \pi^{\mp}$ correlations with $p \bar{p}$ collisions at $\sqrt{s}$ $=1.8 \mathrm{TeV}$ were published by E735 Collaboration in [10].

The correlations between heavy particles (e.g., neutral gauge bosons $Z^{0} Z^{0}$ ) of Bose-Einstein type have not been carried out previously at hadron colliders. Such a study can be addressed to the Large Hadron Collider (LHC) which provide protonproton interactions at $\sqrt{s}=14 \mathrm{TeV}$ centre-of-mass system (c.m.s.) energy.

In this work, we make an attempt to demonstrate that the problem of properties of the genuine interactions can be explored using experimental data which can be collected by ATLAS and CMS Collaborations at the LHC. These data can be analyzed through the compared measures of some inclusive distributions and final state correlations.

One of the aims of this paper is to carry out the proposal for the experimental measurements of the $Z^{0} Z^{0}$ pair correlations.

This exploration will be theoretically supported by the quantum field theory at finite temperature $\left(Q F T_{\beta}\right)$ model approach [3]. It is known that the effective temperature of the vacuum or the ground state or even the thermalized state of particles distorted by external forces is occurring in models quantized in external fields. One of the main parameters of the model is the temperature of the particle source under the 
random source operator influence. The main channels are the di-lepton production $p p \rightarrow Z^{0} Z^{0} \rightarrow 2 e^{-} 2 e^{+}, 2 \mu^{-} 2 \mu^{+}, e^{-} e^{+} \mu^{-} \mu^{+}$in $p p$ collisions.

An efficient selection of leptons needs to be performed according to the following criteria. First, all leptons were required to lie in the pseudorapidity range covered by, e.g., the CMS muon system that is, $|\eta| \leq 2.4$. Second, the leptons were required to be unlikely charged in pairs. Note that the acceptances of another multipurpose detector ATLAS in the azimuthal angle and pseudorapidity are close to the respective parameters of CMS.

The dilepton channel is especially promising from the experimental point of view, since it is expected that the experimental facilities related for LHC (CMS and ATLAS detectors) will make it possible to record muons of energy in the $\mathrm{TeV}$ range with a resolution of about a few percent and an efficiency close to $100 \%$. Moreover, this channel is characterized by a maximum signal-to-background ratio in the energy region being considered.

\section{BEC in case of two particles}

A pair of identical bosons with the mass $m$ produced incoherently (in ideal nondisturbed, noninteracting cases) from an extended source will have an enhanced probability $C_{2}\left(p_{1}, p_{2}\right)=N_{12}\left(p_{1}, p_{2}\right) /\left[N_{1}\left(p_{1}\right) \cdot N_{2}\left(p_{2}\right)\right]$ to be measured in terms of differential cross section $\sigma$, where

$$
N_{12}\left(p_{1}, p_{2}\right)=\frac{1}{\sigma} \frac{d^{2} \sigma}{d \Omega_{1} d \Omega_{2}}
$$

to be found close in 4-momentum space $\Re_{4}$ when detected simultaneously, as compared to if they are detected separately with

$$
N_{i}\left(p_{i}\right)=\frac{1}{\sigma} \frac{d \sigma}{d \Omega_{i}}, d \Omega_{i}=\frac{d^{3} \vec{p}_{i}}{(2 \pi)^{3} 2 E_{p_{i}}}, E_{p_{i}}=\sqrt{\vec{p}_{i}^{2}+m^{2}}, \quad i=1,2 .
$$

On the other hand, the following relation can be used to retrieve the BEC function $C_{2}(Q)$ :

$$
C_{2}(Q)=\frac{N(Q)}{N^{r e f}(Q)},
$$

where $N(Q)$ in general case refer to the numbers for neutral gauge bosons (eg., $Z^{0} Z^{0}$ ) with

$$
Q=\sqrt{-\left(p_{1}-p_{2}\right)_{\mu} \cdot\left(p_{1}-p_{2}\right)^{\mu}}=\sqrt{M^{2}-4 m^{2}} .
$$

In definitions (3) and (4), $N^{\text {ref }}$ is the number of particle pairs without BEC and $p_{\mu_{i}}=\left(\omega_{i}, \vec{p}_{i}\right)$ are four-momenta of produced bosons $(i=1,2) ; M=\sqrt{\left(p_{1}+p_{2}\right)_{\mu}^{2}}$ is the invariant mass of the pair of bosons.

An essential problem in extracting the correlation is the estimate of the reference distribution $N^{r e f}(Q)$ in Eq. (3). If there are other correlations beside the BoseEinstein effect, the distribution $N^{r e f}(Q)$ should be replaced by a reference distribution corresponding to the two-particle distribution in a geometry without BEC. Hence, the expression (3) represents the ratio between the number of $Z^{0} Z^{0}$ pairs $N(Q)$ in the 
real world and the reference sample $N^{r e f}(Q)$ in the imaginary world. Note that the reference sample can not be directly observed in an experiment. Different methods are usually applied for the construction of reference samples [1], however all of them have strong restrictions. One of the preferable methods is to construct the reference samples directly from data. For our aim for reference sample $N^{r e f}(Q)$, it is suitable to use the pairs $Z^{0} Z^{0}$ from different (mixed) events.

It is commonly assumed that the maximum of two-particle BEC function $C_{2}(Q)$ is 2 for $\vec{p}_{1}=\vec{p}_{2}$ if no any distortion and final state interactions are taking into account.

There are experimental difficulties in a determination of $Z^{0} Z^{0}$ correlations, which are associated with acceptance limitations and limited statistics in the $Z^{0} Z^{0}$ sample.

In general, the shape of the BEC function $C_{2}(Q)$ is model dependent. The most simple form of Goldhaber-like parameterization for $C_{2}(Q)$ [11] has been used for data fitting:

$$
C_{2}(Q)=C_{0} \cdot\left(1+\lambda e^{-Q^{2} R^{2}}\right) \cdot(1+\varepsilon Q),
$$

where $C_{0}$ is the normalization factor, $\lambda$ is the so-called the chaoticity strength factor, meaning $\lambda=1$ for fully incoherent and $\lambda=0$ for fully coherent sources; the parameter $R$ is interpreted as a radius of the particle source, often called as the "correlation radius", and assumed to be spherical in this parameterization. The linear term in (5) is supposed to be account within the long-range correlations outside the region of BEC. Note that distribution of bosons can be either far from isotropic, usually concentrated in some directions or almost isotropic, and what is important that in both cases the particles are under the random chaotic interactions caused by other fields in the thermal bath. In the parameterization (5) all of these problems are embedded in the random chaoticity parameter $\lambda$. To advocate the formula (5) it is assumed:

a. incoherent average over particle source where $\lambda$ serve to account for:

- partial coherence,

- long-lived resonances associated with multiple distinguishable sources,

- $Z^{0} Z^{0}$ purity;

b. spherical Gaussian density of particle emission cell (with radius $R$ );

c. static source which means no time (energy) dependence.

In order to save the quantum pattern of particle production process and to avoid the static and undistorted character of particle emitter source we also suggest to use the $C_{2}(Q)$ function within $Q F T_{\beta}$ accompanying by quantum evolution approach in the form:

$$
C_{2}(Q)=\xi(N) \cdot\left[1+\frac{1}{(1+\alpha)\left(1+\alpha^{\prime}\right)} \tilde{\Omega}(Q)+\frac{2 \sqrt{\alpha \alpha^{\prime}}}{(1+\alpha)\left(1+\alpha^{\prime}\right)} \sqrt{\tilde{\Omega}(Q)}\right] \cdot F(Q, \Delta x)
$$

where $\xi(N)$ depends on the multiplicity $N$ as

$$
\xi(N)=\frac{\langle N(N-1)\rangle}{\langle N\rangle^{2}} .
$$

The function $F(Q, \Delta x)$ that expresses the correlation magnitude as a function of $Q$ and two-particle relative distance $\Delta x$ is a consequence of the Bogolyubov's principle 
of correlations weakening at large distances [12]

$$
F(Q, \Delta x)=\frac{f(Q, \Delta x)}{f\left(p_{1}\right) \cdot f\left(p_{2}\right)}=1+r_{f} Q+\ldots
$$

The function (8) is normalized as $F(Q, \Delta x=\infty)=1$, and $r_{f}$ is the measure of correlations weakening where $r_{f} \rightarrow 0$ as $\Delta x \rightarrow \infty ; f(Q, \Delta x)$ is the two-particle distribution function with $\Delta x$, while $f\left(p_{i}\right)$ are one-particle probability functions with $i=1,2$.

The important parameter $\alpha$ (as well as $\alpha^{\prime}$ ) in (6) summarizes our knowledge of other than space-time characteristics of the particle emitting source, and plays the role of a coherence parameter (see [4] for details).

The $\tilde{\Omega}(q)$ in (6) has the following structure in momentum space

$$
\tilde{\Omega}(Q)=\Omega(Q) \cdot \gamma(n)
$$

where

$$
\Omega(Q)=\exp \left(-\Delta_{p \Re}\right)=\exp \left[-\left(p_{1}-p_{2}\right)^{\mu} \Re_{\mu \nu}\left(p_{1}-p_{2}\right)^{\nu}\right]
$$

is the smearing smooth dimensionless generalized function, $\Re_{\mu \nu}$ is the (nonlocal) structure tensor of the space-time size (BEC formation domain), and it defines the spherically-like domain of emitted (produced) bosons.

To clarify with $\gamma(n)$ in (9) let us emphasize that most of experiments dealing with elementary particles at high energies are of an inclusive as one measures quantum effect of BEC on limited samples of particles produced only. The unobserved part of the rest particle system acts then as a kind of thermal (heat) bath influencing measured samples of data (observables). Actually, the temperature $T$ being the most important parameter describing the influence of such a thermal bath is occurred in this model.

The function $\gamma(n)$ reflects the quantum thermal features of BEC pattern and is defined as

$$
\gamma(n)=\frac{n^{2}(\bar{\omega})}{n(\omega) n\left(\omega^{\prime}\right)}, \quad n(\omega) \equiv n(\omega, \beta)=\frac{1}{e^{(\omega-\mu) \beta}-1}, \quad \bar{\omega}=\frac{\omega+\omega^{\prime}}{2},
$$

where $n(\omega, \beta)$ is the mean value of quantum numbers for Bose-Einstein statistics particles with the energy $\omega$ and the chemical potential $\mu$ in the thermal bath with statistical equilibrium at the temperature $T=1 / \beta$. The following condition $\sum_{f} n_{f}(\omega, \beta)=N$ is evident, where the discrete index $f$ reflects the one-particle state $f$.

In terms of time-like $R_{0}$, longitudinal $R_{L}$ and transverse $R_{T}$ components of the space-time size $R_{\mu}$ the distribution $\Delta_{p \Re}$ looks like:

$$
\Delta_{p \Re} \rightarrow \Delta_{p R}=\left(\Delta p^{0}\right)^{2} R_{0}^{2}+\left(\Delta p^{L}\right)^{2} R_{L}^{2}+\left(\Delta p^{T}\right)^{2} R_{T}^{2} .
$$

Seeking for simplicity one has $\left(R_{L}=R_{T}=R\right)$

$$
\Delta_{p R}=\left(p_{1}^{0}-p_{2}^{0}\right)^{2} R_{0}^{2}+\left(\vec{p}_{1}-\vec{p}_{2}\right)^{2} \vec{R}^{2}
$$

for identical bosons. 
Hence, we have introduced a new parameter $R_{\mu}$, a 4 -vector, which defines the region of nonvanishing particle density with the space-time extension of the particle emission source. Expression (10) must be understood in the sense that $\Omega(Q)$ is a function that in the limit $R \rightarrow \infty$, strictly becomes a $\delta$-function. For practical using with ignoring the energy-momentum dependence of $\alpha$, and assuming that $\alpha^{\prime}=\alpha(\alpha$ is related with $C_{2}(0)$ and $\left.N\right)$, we get the expression with $\Omega(Q) \simeq \exp \left(-Q^{2} R^{2}\right)$ :

$$
C_{2}(Q) \simeq \xi(N)\left\{1+\lambda_{\text {new }}(\beta) e^{-Q^{2} R^{2}}\left[1+\lambda_{\text {corr }}(\beta) e^{+Q^{2} R^{2} / 2}\right]\right\}
$$

where the new intercept function becomes as $\lambda_{\text {new }}=\gamma(\omega, \beta) /(1+\alpha)^{2}$, and the new coherence correction in the brackets of Eq. (14) carries an additional intercept function $\lambda_{\text {corr }}=2 \alpha / \sqrt{\gamma(\omega, \beta)}$. In fact, since $\alpha \neq \alpha^{\prime}$ (because $\omega \neq \omega^{\prime}$ and, therefore, the number of states identified here with the number of particles $n(\omega)$ with given energy is also different), one can use the general precise form (6) for $C_{2}$ with details given by Eqs. (9) and (11) and with $\alpha$ coherence function depending on the particle mass, the energy of final leptons produced in pairs within the decays of $Z^{0}$ 's and such characteristics of the emission process as the temperature $T$ and chemical potential $\mu$ occurring in the definition of $n(\omega)$ in (11).

Since we did not follow special assumptions on the quantum operator level for $C_{2}(Q)$ from the initial stage, it may correspond to a physically real and observable effect at the LHC. This pattern may lead to a new squeezing state of correlation region.

\section{Stochastic field and Green's function}

Let us consider the stochastic field $B_{\mu}(x)=B_{\mu_{\tilde{s}}}(x, \tau)$ that depends on the arbitrary random source $\tilde{s}(x)$, and the fifth component $\tau$ means the "stochastic time". The differential equation of an evolution of the field operator $B_{\mu_{\tilde{s}}}(x, \tau)=B_{\mu}(x, \tau)$ in the system under the associated stochastic process is

$$
\partial_{\tau} B_{\mu}(x, \tau)=O\left[B_{\mu}(x, \tau)\right]
$$

where $O\left[B_{\mu}(x, \tau)\right]$ is the differential stochastic operator which has the form

$$
O\left[B_{\mu}(x, \tau)\right]=-\frac{1}{V} \frac{\delta J\left[B_{\mu}(x, \tau)\right]}{\delta B_{\mu}(x, \tau)}+\tilde{s}_{\mu}(x, \tau)
$$

with a volume $V$ being introduced by dimensional reason. The r.h.s. of Eq. (16) is the so-called stochastic operator where $J=\int d^{4} y L\left[B_{\mu}(y), \partial_{\nu} B_{\mu \nu}(y)\right]$ is the action defined by the Lagrangian density $L ; \tilde{s}_{\mu}(x, \tau)=s_{\mu}(x, \tau)+n_{\mu} P$ carries the random stochastic history where the memory dissipation forces and the heat bath effects are included into $s_{\mu}(x)=s_{\mu}(x, \tau)$, the constant $P$ emerges within the action of the stationary forces. Equation (15) is nothing other but the evolution equation of the Langevin type applied already to stochastic processes on the quantum operator level in derivation of multiparticle Bose-Einstein correlations [3]. 
For simplicity, we assume that $\tilde{s}_{\mu}(x)$ varies stochastically with the Gaussian correlation function

$$
\left\langle\tilde{s}_{\mu}(x) \tilde{s}_{\nu}(y)\right\rangle=\text { const } \delta_{\mu \nu} \exp \left(-z^{2} / l_{c h}^{2}\right),
$$

where $z_{\nu}=(x-y)_{\nu}$, and const is the strength of the noise described by the distribution function $\exp \left(-z^{2} / l_{c h}^{2}\right)$ with $l_{c h}$ being the noise characteristic scale. Both const and $l_{c h}$ define the influence of the (Gaussian) noise on, e.g., correlations between particles that "feel" an action of an environment. Actually, Eq. (15) can be transferred to the standard field equation of motion (in Euclidean space)

$$
\frac{1}{V} \frac{\delta J\left[B_{\mu}(x)\right]}{\delta B_{\mu}(x)}=\tilde{s}_{\mu}(x)
$$

with the source $\tilde{s}_{\mu}(x)$ if both $B_{\mu}$ and $\tilde{s}_{\mu}$ do not depend on "stochastic time" $\tau$. In classical theory, the random process given by $\tilde{s}_{\mu}(x)$ is nothing other but the white (Gaussian) noise.

In this paper, we focus on the role of particle masses and energies, effects of coherence and distortion, and the heat bath influences which are rather important to describe the correlations between particles. To solve this problem, especially to derive the memory term in evolution equation one can use the general properties of $Q F T_{\beta}$. The model is defined by the following generating functional in four-dimensional spacetime

$$
Z=\int D B_{\mu} \exp \left[-i \int d^{4} x L\left(B_{\mu}, B_{\mu \nu}\right)\right]
$$

where

$$
L=-\frac{1}{4} B_{\mu \nu} B^{\mu \nu}+\frac{1}{2}\left(m^{2}+U\right) B_{\mu} B^{\mu}
$$

with $B_{\mu \nu}=\partial_{\mu} B_{\nu}-\partial_{\nu} B_{\mu}$.

The direct calculations using the solution of Eq. (15) with the Lagrangian density (20) leads to the propagator of the field $B_{\mu}(x, \tau)$ distorted by $\tilde{s}_{\mu}(x, \tau)$. The transverse part of $B_{\mu}(x, \tau)$ will give the correct expression for the Euclidean vector field propagator at $\tau \rightarrow \infty$.

We are working with fields that correspond to a thermal field $B_{\mu}(x)$ with the standard definition of the Fourier transformed propagator $F\left[\tilde{G}_{\mu \nu}(p)\right]$

$$
F\left[\tilde{G}_{\mu \nu}(p)\right]=G_{\mu \nu}(x-y)=\operatorname{Tr}\left\{T\left[B_{\mu}(x) B_{\nu}(y)\right] \rho_{\beta}\right\}
$$

with $\rho_{\beta}=e^{-\beta H} / \operatorname{Tr}^{-\beta H}$ being the density matrix of a local system in equilibrium at temperature $T$ under the Hamiltonian $H$

$$
H=\int \frac{d^{3} \vec{p}}{(2 \pi)^{3} 2 p^{0}} p^{0} \sum_{\lambda=1}^{3} b^{\lambda^{+}}(p) b^{\lambda}(p)
$$

with the operators of annihilation $b^{\lambda}(p)$ and creation $b^{\lambda^{+}}(p)$ to be defined later. 
The interaction of $B_{\mu}(x)$ with the external field is given by the potential $U$. The equation of motion is

$$
\left(\nabla^{2}+m^{2}\right) B_{\mu}(x)=-J_{\mu}(x),
$$

where $J_{\mu}(x)=U B_{\mu}(x)$ is the source density operator. A simple model like this allows one to investigate the origin of the unstable state of the thermalized equilibrium in a nonhomogeneous external field under the influence of source density operator $J_{\mu}(x)=U B_{\mu}(x)$. For example, the source can be considered as $\delta$-like generalized function, $J_{\mu}(x)=\tilde{\mu} \rho(x, \epsilon) B_{\mu}(x)$ in which $\rho(x, \epsilon)$ is a $\delta$-like succession giving the $\delta$ function as $\epsilon \rightarrow 0$ (where $\tilde{\mu}$ is some massive parameter). This model is useful because the $\delta$-like potential $U(x)$ provides the conditions for restricting the particle emission domain (or the deconfinement region). We suggest the following form:

$$
J_{\mu}(x)=-J_{\text {sys }}(x) B_{\mu}(x)+J_{R_{\mu}}(x),
$$

where the source $J_{\mu}(x)$ is a sum of a regular systematic motion part $J_{\text {sys }}(x)$ and the random source $J_{R_{\mu}}(x)$. The equation of motion (23) becomes

$$
\left[\nabla^{2}+m^{2}-J_{s y s}\right] B_{\mu}(x)=-J_{R_{\mu}}(x),
$$

and the propagator satisfies the following equation (in the Fourier transformed form labeled by tilde):

$$
\left[p_{\mu}^{2}-m^{2}+\tilde{J}_{s y s}\right] \tilde{G}_{\mu \nu}\left(p_{\mu}\right)=\tilde{d}_{\mu \nu}(p)
$$

where

$$
d_{\mu \nu}(x)=\left(g_{\mu \nu}+\frac{1}{m^{2}} \frac{\partial^{2}}{\partial x_{\mu} \partial x_{\nu}}\right) \delta(x) .
$$

As the standard point, the Green's function of the vector field can be obtained from the one of the scalar field acting by the relevant operator $g_{\mu \nu}+m^{-2} \partial^{2} /\left(\partial x_{\mu} \partial x_{\nu}\right)$.

The solution of Eq. (23) is

$$
B_{\mu}(x)=-\int d y G_{\mu \nu}(x, y) J_{R_{\nu}}(y)
$$

where the Green's function obeys the Eq. (26).

\section{Green's function and kernel operator}

Let us go to the thermal field operator $B_{\mu}(x)$ by means of the linear combination of the frequency parts $B_{\mu}^{1}(x)$ and $B_{\mu}^{2^{+}}(x)$

$$
B_{\mu}(x)=B_{\mu}^{1}(x)+B_{\mu}^{2^{+}}(x)
$$

with $\left[B_{\mu}(x), B_{\nu}(y)\right]=i D_{\mu \nu}(x-y)$ and

$$
B_{\mu}^{1}(x)=\int \frac{d^{3} \vec{p}}{(2 \pi)^{3} 2\left(\vec{p}^{2}+m^{2}\right)^{1 / 2}} \sum_{\lambda=1}^{3} \epsilon_{\mu}^{(\lambda)}(p) \tilde{b}^{(\lambda)}(p) e^{-i p x},
$$




$$
B_{\mu}^{2^{+}}(x)=\int \frac{d^{3} \vec{p}}{(2 \pi)^{3} 2\left(\vec{p}^{2}+m^{2}\right)^{1 / 2}} \sum_{\lambda=1}^{3} \epsilon_{\mu}^{(\lambda)}(p) \tilde{b}^{(\lambda)^{+}}(p) e^{i p x} .
$$

The following properties of polarization vectors are the standard ones:

$$
\begin{gathered}
\epsilon_{\mu}^{(\lambda)}(p) \epsilon_{\mu}^{\left(\lambda^{\prime}\right)}(p)=g_{\lambda \lambda^{\prime}} \\
\sum_{\lambda=1}^{3} \epsilon_{\mu}^{(\lambda)}(p) \epsilon_{\nu}^{(\lambda)}(p)=-g_{\mu \nu}+\frac{p_{\mu} p_{\nu}}{m^{2}} .
\end{gathered}
$$

We assume that the deviation from the asymptotic free state given by the operator $a(\vec{p}, t)$ is provided by the random operator $r(\vec{p}, t): a(\vec{p}, t) \rightarrow b(\vec{p}, t)=a(\vec{p}, t)+r(\vec{p}, t)$. The operators $\tilde{b}^{(\lambda)}(p)$ and $\tilde{b}^{(\lambda)^{+}}(p)$ obey the following equations in $\Re_{4}$ (see details in $[3])$ :

$$
\begin{aligned}
{[\omega-\tilde{K}(p)] \tilde{b}^{(\lambda)}(p) } & =\tilde{F}(p)+\rho\left(\omega_{P}, \epsilon\right), \\
{\left[\omega-\tilde{K}^{+}(p)\right] \tilde{b}^{(\lambda)^{+}}(p) } & =\tilde{F}^{+}(p)+\rho^{*}\left(\omega_{P}, \epsilon\right),
\end{aligned}
$$

where $p_{\mu}=\left(\omega=p^{0}, \vec{p}\right)$. Both equations (30) and (31) can be transformed into new equations for the frequency parts $B_{\mu}^{1}(x)$ and $B_{\mu}^{2^{+}}(x)$

$$
\begin{gathered}
i \partial_{0} B_{\mu}^{1}(x)+\int_{\Re_{4}} K(x-y) B_{\mu}^{1}(y) d y=f_{\mu}(x), \\
-i \partial_{0} B_{\mu}^{2^{+}}(x)+\int_{\Re_{4}} K^{+}(x-y) B_{\mu}^{2^{+}}(y) d y=f_{\mu}^{+}(x),
\end{gathered}
$$

where

$$
\begin{aligned}
& f_{\mu}(x)=\int \frac{d^{3} \vec{p}}{(2 \pi)^{3} 2\left(\vec{p}^{2}+m^{2}\right)^{1 / 2}} \sum_{\lambda=1}^{3} \epsilon_{\mu}^{(\lambda)}(p)\left[\tilde{F}(p)+\rho\left(\omega_{P}, \epsilon\right)\right] e^{-i p x} \\
& f_{\mu}^{+}(x)=\int \frac{d^{3} \vec{p}}{(2 \pi)^{3} 2\left(\vec{p}^{2}+m^{2}\right)^{1 / 2}} \sum_{\lambda=1}^{3} \epsilon_{\mu}^{(\lambda)}(p)\left[\tilde{F}^{+}(p)+\rho^{*}\left(\omega_{P}, \epsilon\right)\right] e^{i p x} .
\end{aligned}
$$

The equations for field components $B_{\mu}^{1}(x)$ and $B_{\mu}^{2^{+}}(x)$ (32) and (33), respectively, are nonlocal within the presence of the formfactors $K(x-y)$ and $K^{+}(x-y)$, respectively. In principle, these formfactors can admit the description of locality for nonlocal interactions. At this stage, it must be stressed that we have new generalized evolution equations (32) and (33), which retain the general features of the propagating and interacting of the quantum vector fields with mass $m$ that are in the heat bath (thermal reservoir) and are chaotically distorted by other fields. For further analysis, let us rewrite the system of Eqs. (32) and (33) in the following form:

$$
\begin{gathered}
i \partial_{0} B_{\mu}^{1}(x)+K(x) \star B_{\mu}^{1}(x)=f_{\mu}(x), \\
-i \partial_{0} B_{\mu}^{2^{+}}(x)+K^{+}(x) \star B_{\mu}^{2^{+}}(x)=f_{\mu}^{+}(x),
\end{gathered}
$$


where $A(x) \star B(x)$ is the convoluted function of the generalized functions $A(x)$ and $B(x)$. Applying the direct Fourier transformation to both sides of Eqs. (36) and (37) with the following properties of the Fourier transformation

$$
F\left[K(x) \star B_{\mu}^{i}(x)\right]=F[K(x)] F\left[B_{\mu}^{i}(x)\right]\left(i=1,2^{+}\right),
$$

we get two equations

$$
\begin{gathered}
{\left[p^{0}+\tilde{K}(p)\right] \tilde{B}_{\mu}^{1}(p)=F\left[f_{\mu}(x)\right],} \\
{\left[-p^{0}-\tilde{K}^{+}(p)\right] \tilde{B}_{\mu}^{2^{+}}(p)=F\left[f_{\mu}^{+}(x)\right] .}
\end{gathered}
$$

Finally, we have got the following equation for $\tilde{B}_{\mu}(p)$ field:

$$
\left[-p^{0}+\tilde{K}^{+}(p)\right]\left[p^{0}+\tilde{K}(p)\right] \tilde{B}_{\mu}(p)=\tilde{T}_{\mu}(p),
$$

where

$$
\tilde{T}_{\mu}(p)=\left[-p^{0}+\tilde{K}^{+}(p)\right] F\left[f_{\mu}(x)\right]+\left[p^{0}+\tilde{K}(p)\right] F\left[f_{\mu}^{+}(x)\right] .
$$

We are now at the stage of the main strategy: one has to identify the field $B_{\mu}(x)$ and the random source operator $J_{R_{\mu}}(x)$, introduced in Eq. (25, with the Fourier transformed field $\tilde{B}_{\mu}(p)$ and $\tilde{T}(p)$ in (40), respectively.

The next step is our requirement that Green's function $\tilde{G}_{\mu \nu}(p)$ in Eq. (26) and the function $\Gamma_{\mu \nu}(p)$, satisfying the equation

$$
\left[-p^{0}+\tilde{K}^{+}(p)\right]\left[p^{0}+\tilde{K}(p)\right] \tilde{\Gamma}_{\mu \nu}(p)=g_{\mu \nu}
$$

must be equal to each other, i.e.

$$
F\left[\tilde{G}_{\mu \nu}(p)-\tilde{\Gamma}_{\mu \nu}(p)\right]=0 .
$$

The kernel operator $\tilde{K}(p)$ is

$$
\tilde{K}(p) \simeq \epsilon \sqrt{1+\frac{m^{2}}{\epsilon^{2}}}
$$

where $\epsilon=2 \sqrt{\vec{k}_{l}^{2}+m_{l}^{2}}$ is the total energy of the final lepton-antilepton pair (with momentum $\vec{k}_{l}$ and the mass $m_{l}$ for the lepton) produced within the decay of $Z^{0}$ boson being in the rest frame. To get $\tilde{K}(p)$ in the form(42) we used the fact that the full Green's function $\tilde{G}_{\mu \nu}(p)$ is given by the corresponding full Green's function of the scalar field [13] under the action by the differential operator $\left(g_{\mu \nu}-m^{-2} p_{\mu} p_{\nu}\right)$.

\section{Source size}

It has been emphasized [4] that there are two different scale parameters in the model considered here. One of them is the so-called "correlation radius" $R$ introduced in (5) and (6) with (12). In fact, this $R$-parameter gives the pure size of the particle emission source without the external distortion and interaction coming from other fields. The other (scale) parameter is the stochastic scale $L_{s t}$ which carries the dependence of the 
particle mass, the $\alpha$-coherence degree and what is very important - the temperature $T$-dependence:

$$
L_{s t} \simeq\left[\frac{1}{\alpha(N)\left|p^{0}-\tilde{K}(p)\right|^{2} n(m, \beta)}\right]^{\frac{1}{2}} \rightarrow\left[\frac{1}{\alpha(N) 4 \vec{k}_{l}^{2}\left|1-\delta_{k}\right|^{2} \bar{n}(m, \beta)}\right]^{\frac{1}{5}},
$$

where

$$
\delta_{k}=\sqrt{1+\frac{m^{2}}{4 \vec{k}_{l}^{2}}}
$$

and the lepton mass $m_{l}$ is neglected.

It turns out that the scale $L_{s t}$ defines the range of stochastic forces. This effect is given by $\alpha(N)$-coherence degree which can be estimated from the experiment within the two-particle BEC function $C_{2}(Q)$ when $Q$ close to zero, $C_{2}(0)$, at fixed value of mean multiplicity $\langle N\rangle$ :

$$
\alpha(N) \simeq \frac{2-\bar{C}_{2}(0)+\sqrt{2-\bar{C}_{2}(0)}}{\bar{C}_{2}(0)-1}, \quad \bar{C}_{2}(0)=C_{2}(0) / \xi(N) .
$$

In formula (43), $\bar{n}(m, \beta)$ is the thermal relativistic particle number density

$$
\bar{n}(m, \beta)=3 \int \frac{d^{3} \vec{p}}{(2 \pi)^{3}} n(\omega, \beta)=3 \frac{\mu^{2}+m^{2}}{2 \pi^{2}} T \sum_{l=1}^{\infty} \frac{1}{l} K_{2}\left(\frac{l}{T} \sqrt{\mu^{2}+m^{2}}\right),
$$

where $K_{2}(\ldots)$ is the modified Bessel function.

The coherence function $\alpha$ is another very important one that summarizes our knowledge of other than space-time characteristics of the particle emission source, and the prediction of $\alpha$ from an experiment is very instructive aim itself. For $\alpha=0$, one actually finds

$$
1<C_{2}(Q)<\xi(N)\left(1+\gamma e^{-Q^{2} R^{2}}\right)
$$

which is nothing other but the Goldhaber parameterization [11] with $0<\gamma<1$ being a free parameter adjusting the observed value of $C_{2}(Q=0)$.

Within our aim to explore the correlation between $Z^{0} Z^{0}$ the scale $L_{s t}$ has the form

$$
L_{s t} \simeq\left[\frac{e^{\sqrt{m^{2}+\mu^{2}} / T}}{12 \alpha(N) \vec{k}_{l}^{2}\left(m^{2}+\mu^{2}\right)^{3 / 4}\left(\frac{T}{2 \pi}\right)^{3 / 2}\left(1+\frac{15}{8} \frac{T}{\sqrt{m^{2}+\mu^{2}}}\right)\left|1-\delta_{k}\right|^{2}}\right]^{\frac{1}{5}},
$$

where the condition $l \beta \sqrt{m^{2}+\mu^{2}}>1$ for any integer $l$ in (45) was taken into account. The only lower temperatures will drive $L_{s t}$ within formula (46) even if $\mu=0$ and $l=1$ with the condition $T<m$.

Note that the condition $\mu<m$ is a general restriction in the relativistic "Bose-like gas", and $\mu=m$ corresponds to the Bose-Einstein condensation. 
For high enough $T$ no $\mu$ - dependence is found for $L_{s t}$ :

$$
L_{s t} \simeq\left[\frac{\pi^{2}}{12 \zeta(3) \alpha(N) \vec{k}_{l}^{2} T^{3}\left|1-\delta_{k}\right|^{2}}\right]^{\frac{1}{5}},
$$

where the condition $T>l \sqrt{m^{2}+\mu^{2}}, l=1,2, \ldots$ is taken into account. The origin of formula (47) comes from

$$
\bar{n}(m, \beta) \rightarrow \bar{n}(\beta) \simeq \frac{3 T^{3}}{\pi^{2}} \zeta(3)
$$

where neither a $Z^{0}$ boson mass nor the $\mu$ - dependence occurred; $\zeta(3)=\sum_{l=1}^{\infty} l^{-3}=$ 1.202 is the zeta-function with the argument 3 .

To be close to the experiment there is necessary to include transverse momenta, where the $Z^{0}$ boson mass $m$, in Eqs. (45), (46), (47) is replaced by the transverse mass $m_{T}=\sqrt{m^{2}+p_{T}^{2}}$.

Actually, the increasing of $T$ leads to squeezing of the domain of stochastic force influence, and $L_{s t}\left(T=T_{0}\right)=R$ at some effective temperature $T_{0}$. The higher temperatures, $T>T_{0}$, satisfy to more squeezing effect and at the critical temperature $T_{c}$ the scale $L_{s t}\left(T=T_{c}\right)$ takes its minimal value. Obviously $T_{c} \sim O(200 \mathrm{GeV})$ defines the phase transition where the chiral symmetry restoration will occur. Since in this phase all the masses tend to zero and $\alpha \rightarrow 0$ at $T>T_{c}$ one should expect the sharp expansion of the region with $L_{s t}\left(T>T_{c}\right) \rightarrow \infty$.

The qualitative relation between $R$ and $L_{s t}$ above mentioned is the only one we can emphasize in order to explain the mass dependence of the source size.

\section{Conclusions}

To summarize: the theoretical proposal for two-particle Bose-Einstein correlation function in case of $Z^{0} Z^{0}$ pairs in $p p$ collisions is carried out for the first time.

The correlations of two bosons in 4-momentum space presented in this paper offer useful and instructive complimentary viewpoints to theoretical and experimental works in multiparticle femtoscopy and interferometry measurements at hadron colliders.

We find the time dependence of correlation function calculated in time-dependent external field provided by the operator $r(\vec{p}, t)$ and the chaotic coherence function $\alpha(m, \beta)$. The result can be compared with the static correlation functions (see, e.g., $[14]$ and the references therein mainly devoted to heavy-ion collisions) and also can be used for experimental data fitting.

The stochastic scale $L_{s t}$ decreases with increasing temperatures slowly at low temperatures, and it decreases rather abruptly when the critical temperature is approached.

Our results first predicted for correlation radius $R$ are both $Z^{0}$ boson mass and lepton energy dependent

$$
R \sim \frac{e^{m / 5 T_{0}}}{\alpha^{1 / 5}\left|\vec{k}_{l}\right|^{2 / 5} m^{3 / 10} T_{0}^{3 / 10}}
$$


for low values of $T_{0}<m$, while for higher temperatures, $T_{0}>\sqrt{m^{2}+\mu^{2}}$, one has

$$
R \sim \frac{1}{\alpha^{1 / 5}\left|\vec{k}_{l}\right|^{2 / 5} T_{0}^{3 / 5}}
$$

The theoretical correlation radius $R$ at temperature $T_{0}$ decreases as $Z^{0}$-boson momentum increases. Both estimations (49) and (50) serve as the first approximation to explain the experimental data at different $\sqrt{s}$ and hence at $T$. We claim that the experimental measuring of $R$ (in $f m$ ) can provide the precise estimation of the effective temperature $T_{0}$ which is the main thermal character in the $Z^{0} Z^{0}$ pair emitter source (given by the effective dimension $R$ ) in the proper leptonic decaying channel $Z^{0} Z^{0} \rightarrow l \bar{l} l \bar{l}$ with the final lepton energy $\sqrt{\vec{k}_{l}^{2}+m_{l}^{2}}$ at given $\alpha$ fixed by $C_{2}(Q=0)$ and $\langle N\rangle$. Actually, $T_{0}$ is the true temperature in the region of multiparticle production with dimension $R=L_{s t}$, because at this temperature it is exactly the creation of two particles $\left(Z^{0} Z^{0}\right)$ occurred, and these particles obey the criterion of BEC.

\section{References}

[1] R.M.Weiner, Phys. Rep. 327 (2000) 249.

[2] R.Hanbury-Brown and R.Q.Twiss, Nature 178 (1956) 1046;

[3] G.A.Kozlov, O.V.Utyuzh and G.Wilk, Phys. Rev. C68 (2003) 024901. G.A.Kozlov, Phys. Rev. C58 (1998) 1188; J. Math. Phys. 42 (2001) 4749 and New J. of Physics 4 (2002) 23.1; G.A.Kozlov, "BEC and the particle mass", hep-ph/0512184 G.A.Kozlov, J. Elem. Part. Phys. Atom. Nucl. 36 (2005) 108; G.A. Kozlov, O. Utyuzh, G. Wilk and Z. Wlodarczyk, "Some forgotten features of the Bose-Einstein correlations", hep-ph/0710.3710.

[4] G.A. Kozlov, "BEC and the particle mass", hep-ph/0512184.

[5] P.Langevin, C. R. Acad. Sci. 146 (1908) 530.

[6] The ALEPH Collab., Eur. Phys. J. C36 (2004) 147; Phys. Lett. B611 (2005) 66.

[7] The DELPHI Collab., Phys. Lett. B379 (1996) 330.

[8] G.Abbiendi et al. (OPAL Collab.), Phys. Lett. B559 (2003) 131.

[9] The ZEUS Collab., Phys. Lett. B583 (2004) 231.

[10] T. Alexopoulos et al., [E735 Collaboration], Phys. Rev. D48 (1993) 1931.

[11] G.Goldhaber et al., Phys. Rev. Lett. 3 (1959) 181; G.Goldhaber et al., Phys. Rev. 120 (1960) 300.

[12] N.N. Bogolyubov, "Quasiaveragies in problems of statistical mechanics", JINR report D-781, JINR, Dubna (1961). 
[13] G.A. Kozlov, "Bose-Einstein correlations and the stochastic scale of light hadrons emitter source", hep-ph/arXiv:0801.2072.

[14] C.Y. Wong, W.N. Zhang, Phys. Rev. C76 (2007) 034905. 